\title{
Análise bibliométrica sobre estudo de caso e Survey voltado a inteligência competitiva na indústria
}

\author{
Bibliometric analysis of case study and Survey focused on competitive \\ intelligence in industry
}

Jean Guilherme Azarias ${ }^{1}$

Aparecido dos Reis Coutinho ${ }^{2}$

Fernando Celso Campos ${ }^{3}$
1 Universidade Metodista de Piracicaba jeangazarias@gmail.com

2 Universidade Metodista de Piracicaba arcoutin@unimep.br

3 Universidade Metodista de Piracicaba fernando.campos@unimep.br

\section{Resumo}

Dada a competitividade nos mercados globais, é crescente a busca por informações que resultem em uma vantagem perante os concorrentes, tal processo para a geração e análise das informações é conhecido como Inteligência Competitiva (IC). O presente artigo tem como objetivo examinar a literatura sobre IC aplicada nas indústrias, contemplando estudos de caso e surveys, possibilitando um entendimento das características gerais que as publicações dessa área apresentam. Uma amostra de publicações foi determinada de acordo com critérios de inclusão e exclusão, considerando publicações entre o período de 1997 e 2017 na Scopus. Para o mapeamento da literatura existente sobre um tema, a análise bibliométrica possibilita entender quantitativamente uma amostra sob diversos aspectos, como ano de publicação, origem, autoria e categorias de assunto. O software VOSviewer foi utilizado para visualização de mapas de cocitação e de conteúdo. O trabalho apresenta uma análise quantitativa da literatura publicada, de forma a proporcionar um referencial teórico para desenvolvimento de futuras pesquisas nessa área.

Palavras-chave: Inteligência competitiva. Estudo de caso. Survey. Análise bibliométrica.

\begin{abstract}
Given the competitiveness in global markets, the search for Information that results in an advantage over competitors is increasing. The process for generation and analysis of information in companies is known as Competitive Intelligence. This study aims to examine the published literature about Competitive Intelligence (CI) on industries, covering case studies or surveys, understanding the general characteristics that the publications of this area present. A sample of publications was determined according to inclusion and exclusion criteria, being considered publications between the period of 1997 and 2017 in the Scopus database. In order to mapping the existing literature on a topic, the bibliometric analysis provides a quantitative understanding of a sample under several aspects, such as year of publication, origin, authorship, and subject categories. The VOSviewer software was used to view content and co-citation network maps. This study shows a quantitative analysis of the published literature in order to provide a theoretical framework for developing further researches in this area.
\end{abstract}

Keywords: Competitive Intelligence. Case study. Survey. Bibliometric analysis. 


\section{Introdução}

A inteligência competitiva (IC) é abordada sob uma variedade de conceitos tendo em vista as diversas áreas de aplicação (Lucas, Café, \& Viera, 2016). A IC pode ser definida de maneira generalista como "uma forma sistemática para a coleta de dados sobre o mercado e as tendências de negócios, de forma a minimizar riscos durante um processo decisório e atingir os objetivos da empresa" (Kahaner, 1997; Teixeira \& Souza, 2013).

Existem ainda outras definições para IC sugeridas por diversos autores, como Fuld (1994) e Herring (1997), porém, uma das definições mais bem aceitas foi realizada pela SCIP - Sociedade para Profissionais de Inteligência Competitiva (apud Ghannay \& Zeineb, 2012) e coloca a IC como "um processo para aumentar a competitividade no mercado por meio de maior compreensão dos concorrentes e próprio ambiente competitivo, pautado por uma ética incontestável”.

Embora seja recente a utilização de IC em alguns setores, como na indústria de construção, existe uma potencial oportunidade para obtenção de vantagem competitiva por um processo de decisão mais eficiente, principalmente no atual contexto de mercados globalizados (Safa et al., 2015).

Em um estudo sobre ferramentas disponíveis para análise competitiva, (Bose, 2008) observou que as empresas, tanto do setor privado quanto público, estavam iniciando seus próprios serviços de IC a fim de proporcionar um respaldo maior para os seus tomadores de decisões e que IC se tornaria uma necessidade para as empresas em um futuro próximo.

A utilização da IC é reconhecida por profissionais e acadêmicos nas mais diversas áreas, sendo difícil encontrar uma indústria que não poderia obter um certo benefício dos seus resultados, seja para fusões, estratégias empresariais, pesquisa e desenvolvimento de produtos, planos de marketing, desenvolvimento de negócios e etc (Safa et al., 2015).

Apesar da importância da IC ser consolidada e aceita, (Lichtenthaler, 2005) afirmou que as empresas geralmente não se baseiam em trabalhos acadêmicos para suas atividades e, para que isso seja mudado, é necessário desenvolver métodos e aplicações direcionados às necessidades reais das empresas. Os estudos de casos e surveys podem constituir uma forma viável de integração entre a indústria e a academia.

Os estudos de caso são opções para a compreensão, de forma flexível e abrangente, de situações da vida real. São comumente utilizados por acadêmicos como forma de estudar com profundidade o comportamento de uma empresa, ou conjunto de empresas, e realizar generalizações. Um estudo de caso pode ser adaptado por várias abordagens diferentes, ajudando pesquisadores a explorar, explicar, descrever, avaliar e teorizar sobre questões complexas em um determinado contexto (Harrison, Birks, Franklin, \& Mills, 2017).

Para a condução de um estudo de caso, Miguel et al. (2012) propõem uma sequência composta por seis etapas, sendo elas: (1) definição de uma estrutura conceitual-teórica; (2) planejamento dos casos; (3) condução do teste piloto; (4) coleta de dados; (5) análise dos dados; (6) geração de relatório.

No artigo intitulado "Extracting and evaluating conversational patterns in social media: a sócio-semantic analysis of customers' reactions to the launch of new product using Twitter streams" (Lipizzi, Iandoli, \& Ramirez Marquez, 2015), os autores realizaram um estudo de caso analisando as percepções do público em uma rede social após o lançamento de dois produtos, concluindo que esse tipo de análise pode resultar em informações importantes sobre o futuro sucesso do produto no mercado. 
No mesmo sentido, os levantamentos do tipo survey constituem uma abordagem metodológica que permite ao pesquisador avaliar uma amostra significativa de uma situação, extraindo informações que possibilite expandir os resultados para a população ou tirar conclusões apenas para os elementos participantes da pesquisa (Miguel et al., 2012).

Semelhante à sequência de condução do estudo de caso, as surveys são conduzidas por meio de questionários aplicados à uma amostra de indivíduos. A utilização de técnicas estatísticas está diretamente ligada aos procedimentos de survey, como nas etapas de seleção da amostra, tratamento dos dados e análise dos resultados (Forza, 2002).

Como exemplo de survey aplicado em estudos de IC na indústria, o artigo "Perceived environmental uncertainty and competitive intelligence practices” (Yap, Rashid, \& Sapuan, 2013) analisou, por meio da resposta de formulários, como as empresas na Malásia praticam a IC, como percebem as incertezas ambientais e a ligação entre os dois fatores.

De acordo com Zupic e Čarter (2015), o mapeamento científico por meio de análise bibliométrica auxilia pesquisadores a entender a estrutura de um campo de pesquisa. $\mathrm{O}$ uso de métodos quantitativos bibliométricos é importante no contexto cientifico, considerando que o volume de publicações cresce continuadamente e que os pesquisadores encontram cada vez mais dificuldades em rastrear literatura relevante em seu campo de estudo.

Assim, a construção de um referencial teórico por meio de bibliometria proporciona ao pesquisador uma análise quantitativa das publicações, assim como uma visão geral da literatura em estudo, encontrando relações entre os elementos estruturais da amostra, como referências, autores, palavras-chave e região (Zupic \& Čater, 2015).

Nesse contexto, o objetivo geral deste trabalho é explorar as publicações realizadas com te- mas direcionados a estudos de casos ou surveys sobre IC em indústrias, identificando por meio de análise bibliométrica as características gerais e os destaques da literatura.

\section{Procedimento metodológico}

A abordagem metodológica empregada no presente trabalho consiste de uma revisão quantitativa da literatura, realizada por meio de técnicas de análise bibliométrica. Primeiramente, foi pesquisado "competitive intelligence" ("Inteligência Competitiva”) em título, resumo ou palavra-chave de publicações na base de dados Scopus, que apresenta uma ampla cobertura para algumas áreas de pesquisa (Zupic \& Čater, 2015), como as engenharias.

A partir da busca inicial, a limitação do período de tempo foi estabelecida, dando continuidade em uma série de buscas, seguindo critérios de inclusão e exclusão, até a obtenção de uma amostra de artigos considerados relevantes na temática em questão, dos quais foram analisados. Os critérios utilizados para a seleção dos artigos abrangem artigos e trabalhos de conferência, em inglês, aplicados na indústria, excluindo os estudos que citam no título a aplicação em serviços.

Foi utilizado o software VOSviewer versão 1.6.6, de acesso livre, para construção e visualização de mapas bibliométricos, que contemplam os dados dos autores, periódicos, quantidade de citações, referências, palavras-chaves, entre outros (van Eck \& Waltman, 2010). A análise do conteúdo foi feita mediante análise de frequência das palavras, por meio dos conjuntos de termos compartilhados entre os documentos (Delecroix \& Epstein, 2004; Geng et al., 2017).

Além disso, foi analisada a relevância das publicações baseando-se tanto na quantidade de citações na base de dados Scopus, quanto no 
índice de fator de impacto (FI) dos periódicos, segundo o Journal Citation Report de 2015. Foram utilizadas abreviações para melhor disposição dos dados, que são apresentadas na Tabela 1.

Tabela 1: Descrição das abreviações

\begin{tabular}{c|c}
\hline Abreviações & Descrição \\
\hline$A P$ & Ano da publicação do artigo \\
\hline FI & Fator de impacto do periódico \\
\hline$M C$ & Média de citações por ano \\
\hline$Q C$ & Quantidade de citações \\
\hline$Q P$ & Quantidade de publicações \\
\hline
\end{tabular}

\section{Resultados}

Inicialmente, a busca realizada apenas com o termo "Competitive Intelligence" ("Inteligência Competitiva") e sem limitação de tempo retornou um resultado que permite observar crescimento nas publicações a partir do final da década de 1990, conforme ilustrado na Figura 1. Dessa forma, as demais buscas passaram a ser limitadas apenas para publicações contidas entre os anos de 1997 e 2017, abrangendo a maioria dos documentos encontrados.
$\mathrm{Na}$ sequência, foram realizadas buscas com as palavras-chave que caracterizam o objeto do estudo, associando os resultados e aplicando critérios de exclusão para a obtenção de uma amostra pertinente para análise, conforme a Tabela 2.

Tabela 2: Critérios para seleção da amostra final

\begin{tabular}{c|c|c|c}
\hline Busca & Filtros & Pesquisa & Resultados \\
\hline 1 & $\begin{array}{c}\text { Título, resumo ou } \\
\text { palavra-chave }\end{array}$ & $\begin{array}{c}\text { "Competitive } \\
\text { intelligence" }\end{array}$ & 5.671 \\
\hline 2 & $\begin{array}{c}\text { Período de 1997 a } 2017 \\
\text { Apenas palavra-chave }\end{array}$ & $\begin{array}{c}\text { "Competitive } \\
\text { intelligence" }\end{array}$ & 5.248 \\
\hline 3 & $\begin{array}{c}\text { Período de 1997 a 2017 } \\
\text { Título, resumo ou } \\
\text { palavra-chave }\end{array}$ & "Industry" & 1.019 .964 \\
\hline 5 & $\begin{array}{c}\text { Período de 1997 a 2017 } \\
\text { Título, resumo ou } \\
\text { palavra-chave }\end{array}$ & $\begin{array}{c}\text { "Case study" } \\
\text { ou "Survey" }\end{array}$ & 1.782 .057 \\
\hline 6 & - & $\begin{array}{c}\text { Busca 2 + } \\
\text { Busca 3 }\end{array}$ & 1.495 \\
\hline 7 & $\begin{array}{c}\text { Apenas artigos e } \\
\text { conferências } \\
\text { Apenas em inglês }\end{array}$ & $\begin{array}{c}\text { Busca 2 + Busca 2 + } \\
\text { Busca 4 }\end{array}$ & 665 \\
\hline 8 & $\begin{array}{c}\text { Análise do título } \\
\text { Apenas artigos disponíveis }\end{array}$ & Busca 7 & 93 \\
\hline
\end{tabular}

Finalmente, após a conclusão das buscas e aplicação dos critérios, uma amostra de 93 publicações foi selecionada para análise. A Tabela 3 apresenta a quantidade de publicações distribuídas ao longo dos anos.

Apesar de a pesquisa abranger o período de 1997 a 2017, as publicações sobre estudos de casos ou survey de IC na indústria começaram a ocorrer apenas em 2001, tendo seu pico de publicações nos anos de 2006, 2007 e 2008. Até a data de realização da pesquisa não foram encontradas publicações no ano de 2017, assim como no ano de 2009 não houve publicação.
Figura 1: Distribuição da busca inicial sobre competitive intelligence (Inteligência Competitiva) 
Tabela 3: Distribuição das publicações da amostra final ao longo dos anos

\begin{tabular}{c|c}
\hline AP & QP \\
\hline 2001 & 4 \\
\hline 2002 & 8 \\
\hline 2003 & 6 \\
\hline 2004 & 2 \\
\hline 2005 & 4 \\
\hline 2006 & 18 \\
\hline 2007 & 17 \\
\hline 2008 & 10 \\
\hline 2009 & 0 \\
\hline 2010 & 2 \\
\hline 2011 & 1 \\
\hline 2012 & 2 \\
\hline 2013 & 8 \\
\hline 2014 & 6 \\
\hline 2015 & 4 \\
\hline 2016 & 1 \\
\hline
\end{tabular}

\subsection{Distribuição por categorias de assunto}

As publicações encontradas foram classificadas na base de dados Scopus em 14 categorias de assuntos diferentes, podendo haver publicações que abordam mais de um assunto. Observando as principais categorias que ocorrem, conforme a Figura 2, mais da metade delas estão relacionadas

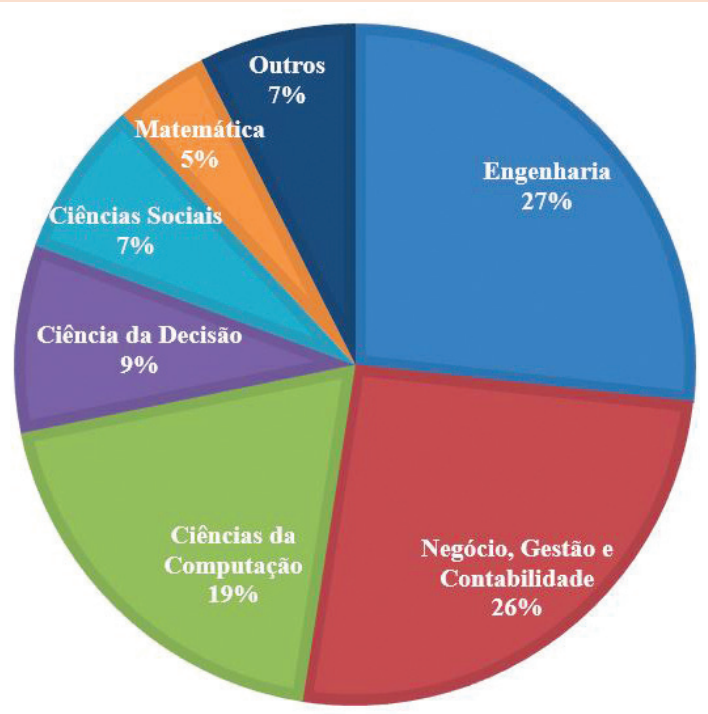

Figura 2: Distribuição por categorias de assuntos

\subsection{Análise da origem}

Os artigos foram analisados quanto à sua origem geográfica, institucional e fonte de publicação. No que diz respeito à origem geográfica, foram encontrados 34 países diferentes, sendo que $90 \%$ das publicações são oriundas de apenas 12 países, conforme Figura 3. Os Estados Unidos aparecem com a maior presença nas publicações, 28 no total, seguido de Austrália e China, ambos com 11 publicações, e Reino Unido com 9. à Engenharia $(27 \%)$ e Negócio, Gestão e Contabilidade (26\%). As Ciências da Computação e da Decisão também aparecem com grande representatividade.

A análise das publicações por categorias de assunto traduz o objetivo dos estudos de caso e surveys sobre IC nas indústrias, que é proporcionar uma melhor gestão do negócio com maior assertividade nas tomadas de decisões, valendo-se na maioria das vezes de sistemas informatizados.

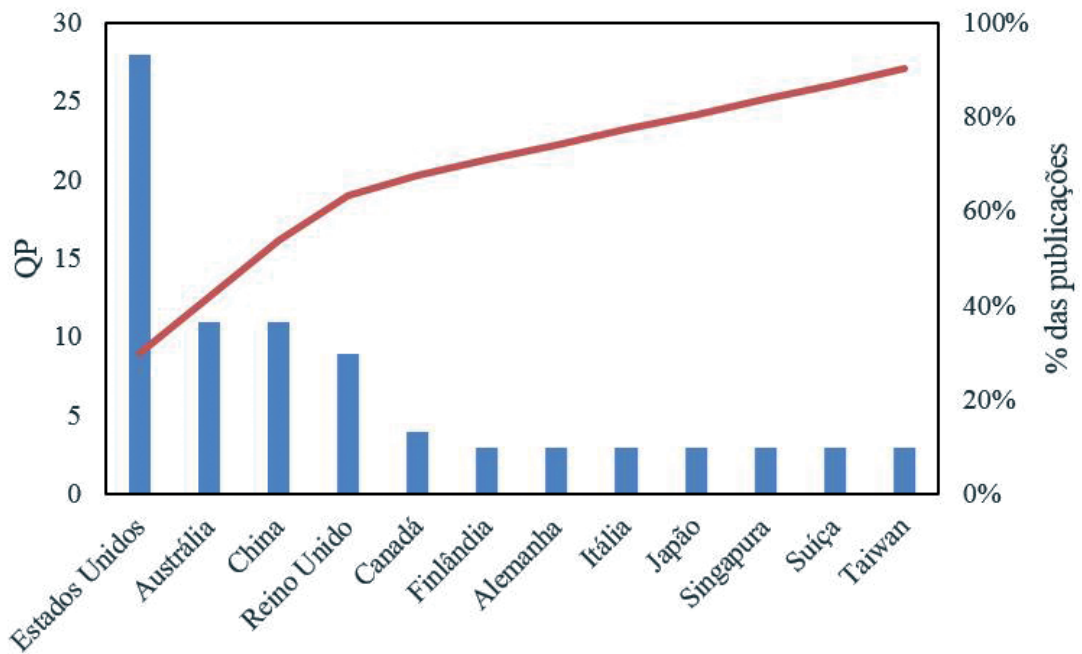

País 
Baseado nas análises de origem, mais de $60 \%$ das publicações são provenientes de apenas quatro países, Estados Unidos, Austrália, China e Reino Unido, todos com força econômica representativa.

Foram identificadas 196 instituições diferentes entre as publicações, sendo que apenas 11 possuem mais de uma publicação. As três universidades que mais se destacam são Monash University - Austrália, com quatro publicações, seguido de Hong Kong Polytechnic University - China e University of Melbourne - Austrália, ambas com três publicações. Observa-se que, esmo os Estados Unidos possuindo a maior representatividade em QP na amostra, nenhuma instituição americana aparece entre as três principais.

Em relação à fonte de publicação, foram encontrados 65 periódicos ou congressos diferentes e, assim como ocorre para as instituições, existe uma grande dispersão das publicações. A fonte de publicação que mais se destaca é o periódico International Journal of Information Management, com seis publicações da amostra e um FI de 2.692 em 2015. Estão representados na Figura 4 os seis principais periódicos em termos de QP, eixo vertical principal, e seus respectivos FI, eixo vertical secundário.

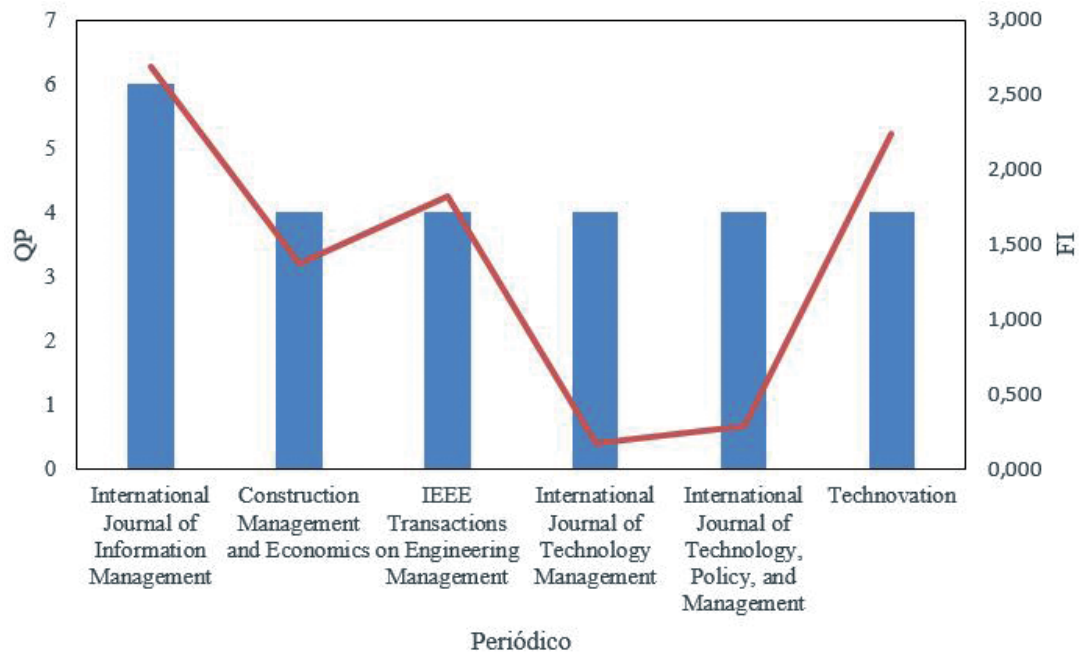

Figura 4: Distribuição de publicações pelos seis principais periódicos

\subsection{Análise de cocitação}

Por meio de uma análise de cocitação, na qual os autores citados pelas 93 publicações da amostra são considerados, foram encontrados 3.963 autores diferentes. A análise considera que quando dois autores são citados pelo mesmo artigo, eles possuem uma ligação e o conjunto dessas ligações foram as correntes de autores.

Foi estabelecido um limite de no mínimo sete citações, que é um valor com representatividade considerável dentro de uma amostra de 93 artigos e ainda assim permite uma quantidade de autores aceitável para formação das correntes.

A Figura 5 ilustra as redes formadas por 33 autores divididos em 4 correntes diferentes, distintas por cores. A corrente principal, representada pela cor vermelha, possui 16 autores e indica que a força de ligação entre esses autores é mais intensa do que nas demais correntes. Em relação à relevância dos autores, quanto maior a circunferência, maior a QC.

No que diz respeito aos principais autores citados pela amostra analisada, nota-se autores da área de gestão estratégica (Barney, 1991; Porter, 1980), metodologia de pesquisa (Eisenhardt, 1989; Yin, 2017), gestão do conhecimento (Nonaka, 2000) e sistemas de informação (Brynjolfsson, 1993; Mylopoulos, Chung, \& Nixon, 1992).

\subsection{Análise do conteúdo da amostra}

O recurso de mapa foi utilizado para analisar o conteúdo das publicações, considerando as palavras que ocorrem no título ou resumo com contagem binária, em que se verifica a ocorrência na publicação, não importando a frequência. Foram encontradas 2.447 palavras diferentes. A 


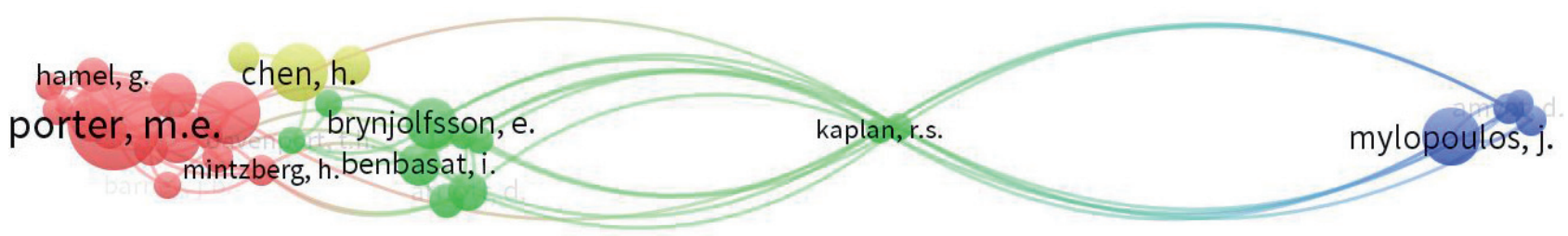

Figura 5: Mapa de rede dos principais autores cocitados

Figura 6 apresenta o mapa das palavras que ocorrem em pelo menos 10 publicações, resultando em um total de 34 palavras.

A rede principal (vermelha) contempla publicações com foco no desempenho competitivo das empresas, a rede secundária (verde) aborda estudos com relação mais próxima das estratégias organizacionais e a terceira corrente (azul) trata da IC relacionada aos sistemas de informação.

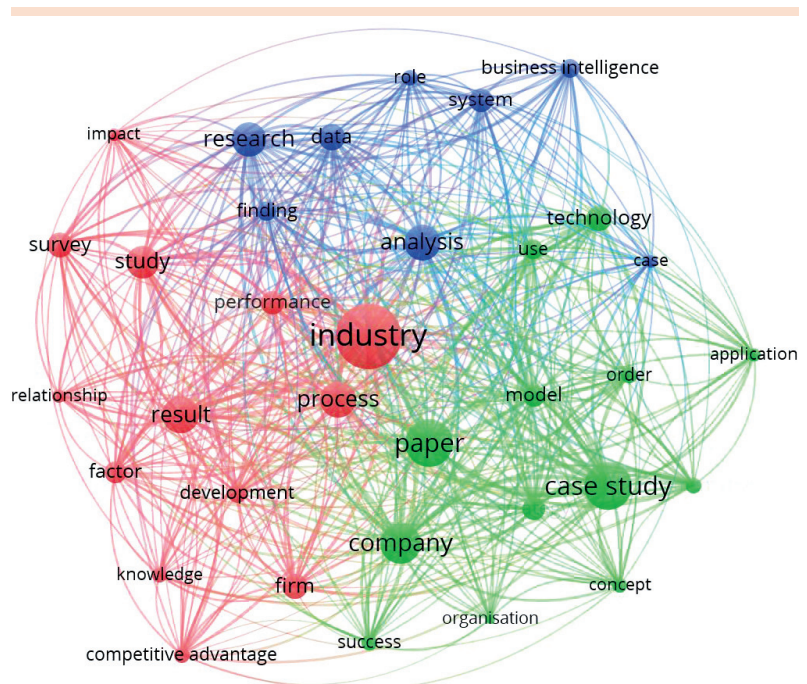

Figura 6: Mapa de coocorrência de palavras

A análise do conteúdo reafirma a estreita relação entre IC e gestão estratégica observada pelos autores em destaque. A presença de palavras como result (resultado), performance (desempenho) e success (sucesso) evidencia que a IC foca na obtenção de uma vantagem competitiva em relação aos concorrentes, enquanto as palavras data (dados), technology (tecnologia) e systems (sistemas) representam os principais meios para a condução da
IC. Tais palavras também são confirmadas pelo conteúdo das publicações mais citadas no período.

No que diz respeito ao método de pesquisa utilizado, a apresenta a distribuição das publicações entre as pesquisas que utilizam o Estudo de caso, Survey ou ambos.

\begin{tabular}{c|c|c}
\hline Método de pesquisa & Quantidade & $\begin{array}{c}\text { Porcentagem } \\
\text { da amostra }\end{array}$ \\
\hline Estudo de caso & 56 & $60,21 \%$ \\
\hline Survey & 30 & $32,26 \%$ \\
\hline $\begin{array}{c}\text { Estudo de caso e } \\
\text { Survey }\end{array}$ & 7 & $7,53 \%$ \\
\hline
\end{tabular}

Tabela 4: Distribuição da amostra pelo método de pesquisa

\subsection{Publicações mais citadas}

A Tabela 4 mostra as cinco publicações mais citadas da amostra em estudo, incluindo a MC, nome do periódico e país de origem do primeiro autor. Os Estados Unidos ocupam a posição de maior relevância, sendo o país de origem de três dentre as cinco publicações, tendo ainda uma publicação da Austrália e uma de Portugal.

A primeira publicação da lista dos mais citados foi publicada no periódico Information and Management, sob o título de "An empirical study of best practices in virtual teams" (Lurey \& Raisinghani, 2001), que trata de um estudo realizado por meio survey para entender como as equipes virtuais são organizadas e a sua efetividade para a geração de vantagem competitiva para a empresa.

Além disso, as demais publicações abordam temas como os aspectos essenciais para tomada 
de decisões efetivas (Arnott \& Pervan, 2008), a utilização de sistemas de informação (JardimGoncalves, Grilo, \& Steiger-Garcao, 2006) e práticas de manufatura ambientalmente sustentáveis (Rusinko, 2007).

$\mathrm{O}$ artigo intitulado "A study of RひD, innovation, and business performance in the Canadian biotechnology industry" (Hall \& Bagchi-Sen, 2002) apresenta a forma que empresas canadenses avaliam a pesquisa e desenvolvimento e a inovação em relação ao seu desempenho no ambiente competitivo.

Como observado anteriormente, o pico na quantidade de publicações da amostra ocorre entre os anos de 2006 e 2008, responsável por 48,39\% das publicações, mesmo período de publicação de três dentre os cinco mais citados.

\section{Conclusão}

Foi realizada uma análise bibliométrica a partir de uma amostra com 93 publicações, entre artigos e trabalhos de conferência, abrangendo es- tudos de caso e surveys sobre IC em indústrias, o que permitiu observar uma forte ligação entre IC e gestão estratégica.

A importância da IC como uma fonte de vantagem competitiva nas empresas é justificada pela possibilidade de obter resultados a partir de um conjunto de informações estruturadas. Entretanto, observa-se uma redução nos estudos nessa área nos últimos anos, podendo ser resultado de estudos em novas áreas, como por exemplo Big Data.

Com base nas categorias de assunto em que a amostra foi classificada na base de dados Scopus, bem como a análise de coocorrência das palavras no título e resumo, é possível identificar os principais assuntos abordados na área, possibilitando um entendimento da literatura em busca de lacunas para futuras pesquisas.

As principais fontes de publicação encontradas na amostra analisada foram o International Journal of Information Management, Construction Management and Economics, IEEE Transactions on Engineering Management, International Journal of Technology Management,

Tabela 5: Os cinco artigos mais citados

\begin{tabular}{|c|c|c|c|c|c|c|c|}
\hline Ano & QC & MC & Autores & Artigo & $\begin{array}{l}\text { Método de } \\
\text { pesquisa }\end{array}$ & Periódico e FI & País \\
\hline 2001 & 223 & 13 & $\begin{array}{l}\text { LUREY, J. S.; } \\
\text { RAISINGHANI, } \\
\text { M. S. }\end{array}$ & $\begin{array}{l}\text { An empirical study of best } \\
\text { practices in virtual teams }\end{array}$ & Survey & $\begin{array}{c}\text { Information and } \\
\text { Management } \\
(3,317)\end{array}$ & $\begin{array}{l}\text { Estados } \\
\text { Unidos }\end{array}$ \\
\hline 2008 & 176 & 19 & $\begin{array}{l}\text { ARNOTT, D.; } \\
\text { PERVAN, G. }\end{array}$ & $\begin{array}{l}\text { Eight key issues for the decision } \\
\text { support systems discipline }\end{array}$ & $\begin{array}{l}\text { Estudo de } \\
\text { caso }\end{array}$ & \begin{tabular}{|c|} 
Decision \\
Support \\
Systems $(3,222)$ \\
\end{tabular} & Austrália \\
\hline 2006 & 113 & 10 & \begin{tabular}{|c|} 
JARDIM- \\
GONÇALVES, \\
R.; GRILO, A.; \\
STEIGER-GARÇÃ̃O, \\
A. \\
\end{tabular} & $\begin{array}{c}\text { Challenging the interoperability } \\
\text { between computers in industry } \\
\text { with MDA and SOA }\end{array}$ & $\begin{array}{l}\text { Estudo de } \\
\text { caso }\end{array}$ & $\begin{array}{l}\text { Computers in } \\
\text { Industry }(2,691)\end{array}$ & Portugal \\
\hline 2007 & 112 & 11 & RUSINKO, C. A. & $\begin{array}{l}\text { Green manufacturing: And } \\
\text { evaluation of environmentally } \\
\text { sustainable manufacturing } \\
\text { practices and their impact on } \\
\text { competitive outcomes }\end{array}$ & Survey & $\begin{array}{c}\text { IEEE } \\
\text { Transactions } \\
\text { on Engineering } \\
\text { Management } \\
(1,940)\end{array}$ & $\begin{array}{l}\text { Estados } \\
\text { Unidos }\end{array}$ \\
\hline 2002 & 96 & 6 & $\begin{array}{l}\text { HALL, L. A.; } \\
\text { BAGCHI-SEM, S. }\end{array}$ & $\begin{array}{l}\text { A study of R\&D, innovation, and } \\
\text { business performance in the } \\
\text { Canadian biotechnology industry }\end{array}$ & Survey & $\begin{array}{l}\text { Technovation } \\
\quad(3,265)\end{array}$ & $\begin{array}{l}\text { Estados } \\
\text { Unidos }\end{array}$ \\
\hline
\end{tabular}


International Journal of Technology, Policy, and Management e Technovation. Assim, esses periódicos em destaque constituem um caminho simplificado para pesquisadores buscarem as informações pertinentes em futuros trabalhos na área.

Existem 16 autores que apresentam maior influência na literatura, sendo M. E. Porter o principal deles. O país com maior número de publicações são os Estados Unidos, enquanto a instituição mais relevante é a Monash University, na Austrália. O mapeamento de onde estão ocorrendo as pesquisas na área, seja pela identificação dos principais autores, países ou instituições, contribui para o encorajamento de futuros trabalhos de colaboração internacional.

Esse estudo possui limitações no método, tendo em vista que foi utilizada apenas uma base de dados na análise e, apesar da Scopus ser uma base de dados importante e com ampla cobertura, pode ocorrer a ausência de trabalhos relevantes na área. Entretanto, a utilização da análise de cocitação considera também as publicações não indexadas na Scopus, mitigando parcialmente essa limitação.

Ainda, a utilização de uma abordagem quantitativa proporciona uma limitação na análise da literatura. Assim, como sugestão para futuras análises, seria interessante a expansão da pesquisa para outras bases de dados e com maior profundidade na análise de conteúdo das publicações.

Considerando os objetivos da IC de proporcionar respaldo para as tomadas de decisões e melhorar a posição competitiva das empresas, para sugestão de futuros trabalhos, é importante o desenvolvimento de estudos empíricos visando estabelecer o grau de correlação entre a utilização da IC nas empresas e o desempenho econômico obtido.

Os resultados do presente trabalho poderão contribuir para que os pesquisadores possam basear suas pesquisas em uma análise quantitativa da literatura já publicada, tendo em vista que a análise bibliométrica permite conhecer os padrões de uma literatura e traçar um caminho para os desdobramentos da pesquisa com base em uma análise quantitativa do referencial teórico.

\section{Agradecimentos}

Ao apoio financeiro disponibilizado pelo programa CAPES/PROSUC.

\section{Referências}

Arnott, D., \& Pervan, G. (2008). Eight key issues for the decision support systems discipline. Decision Support Systems, 44(3), 657-672. https://doi.org/10.1016/j. dss.2007.09.003

Barney, J. (1991). Firm Resources and Sustained Competitive Advantage. Journal of Management, 17(1), 99-120. https://doi.org/10.1177/014920639101700108

Bose, R. (2008). Competitive intelligence process and tools for intelligence analysis. Industrial Management \& Data Systems, 108(4), 510-528. https://doi. org/10.1108/02635570810868362

Brynjolfsson, E. (1993). The productivity paradox of information technology. Communications of the ACM, 36(12), 66-77. https://doi.org/10.1145/163298.163309

Delecroix, B., \& Epstein, R. (2004). Co-word analysis for the non-scientific information example of Reuters Business Briefings. Data Science Journal, 3, 80-87. Retrieved from http://www.jstage.jst.go.jp/article/ dsj/3/0/3_80/_article\%5Cnhttp://www.jstage.jst.go.jp/ article/dsj/3/0/80/_pdf

Eisenhardt, K. M. (1989). Building Theories from Case Study Research. Academy of Manageent Review, 14(4), 532-550. https://doi.org/10.2307/258557

Forza, C. (2002). Survey research in operations management: a process-based perspective. International Journal of Operations \& Production Management, 22(2), 152-194. https://doi. org/10.1108/01443570210414310

Fuld, L. M. (1994). The new competitor intelligence: the complete resource for finding, analyzing, and using information about your competitors. J. Wiley.

Geng, S., Wang, Y., Zuo, J., Zhou, Z., Du, H., \& Mao, G. (2017). Building life cycle assessment research: A review by bibliometric analysis. Renewable and Sustainable Energy Reviews, 76(October 2015), 176184. https://doi.org/10.1016/j.rser.2017.03.068 
Ghannay, J. C., \& Zeineb, B. A. M. (2012). Synergy Between Competitive Intelligence and Knowledge Management: A key for Competitive Advantage. Proceedings of the 13Th European Conference on Knowledge Management, 2, 23-34.

Hall, L. A., \& Bagchi-Sen, S. (2002). A study of R\&amp;D, innovation, and business performance in the Canadian biotechnology industry. Technovation, 22, 231-244. https://doi.org/10.1016/S0166-4972(01)00016-5

Harrison, H., Birks, M., Franklin, R., Mills, J. (2017). Case Study Research: Foundations and Methodological Orientations. Forum: Qualitative Social Research, 18(1). https://doi.org/http://dx.doi.org/10.17169/fqs-18.1.2655

Herring, J. P. (1997). Producing CTI that meets management needs and expectations. In SCIP Competitive Technology Intelligence Symposium. Boston.

Jardim-Goncalves, R., Grilo, A., \& Steiger-Garcao, A. (2006). Challenging the interoperability between computers in industry with MDA and SOA. Computers in Industry, 57(8-9), 679-689. https://doi.org/10.1016/j. compind.2006.04.013

Kahaner, L. (1997). Competitive Intelligence: how to gather, analyze, and use information to move your business to the top. New York: Simon \& Schuster.

Lichtenthaler, E. (2005). The choice of technology intelligence methods in multinationals: towards a contingency approach. International Journal of Technology Management, 32(3/4), 388. https://doi. org/10.1504/IJTM.2005.007341

Lipizzi, C., Iandoli, L., \& Ramirez Marquez, J. E. (2015). Extracting and evaluating conversational patterns in social media: A socio-semantic analysis of customers' reactions to the launch of new products using Twitter streams. International Journal of Information Management, 35(4), 490-503. https://doi.org/10.1016/j. ijinfomgt.2015.04.001

Lucas, A., Café, L. M. A., \& Viera, A. F. G. (2016). Inteligência de negócios e inteligência competitiva na ciência da informação brasileira: contribuições para uma análise terminológica. Perspectivas Em Ciência Da Informação, 21(2), 168-187. https://doi. org/10.1590/1981-5344/2568

Lurey, J. S., \& Raisinghani, M. S. (2001). An empirical study of best practices in virtual teams. Information and Management, 38(8), 523-544. https://doi.org/10.1016/ S0378-7206(01)00074-X

Miguel, P. A. C., Fleury, A. C. C., Mello, C. H. P., Nakano, D. N., Turrioni, J. B., Ho, L. L., ... Pureza, V. M. M. (2012). Metodologia de pesquisa em engenharia de produção e gestão de operações. Rio de Janeiro: Elsevier (ABEPRO). https://doi.org/CDU 658.5001.8
Mylopoulos, J., Chung, L., \& Nixon, B. (1992). Representing and Using Nonfunctional Requirements: A Process-Oriented Approach. IEEE Transactions on Software Engineering, 18(6), 483-497. https://doi. org/10.1109/32.142871

Nonaka, I. (2000). A dynamic theory of organizational knowledge creation. In Knowledge, groupware and the internet (pp. 3-42).

Porter, M. E. (1980). Competitive strategy: techniques for analyzing industries and competitors. New York: Free Press.

Rusinko, C. (2007). Green Manufacturing: An Evaluation of Environmentally Sustainable Manufacturing Practices and Their Impact on Competitive Outcomes. IEEE Transactions on Engineering Management, 54(3), 445-454. https://doi. org/10.1109/TEM.2007.900806

Safa, M., Shahi, A., Haas, C. T., Fiander-McCann, D., Safa, M., Hipel, K., \& MacGillivray, S. (2015). Competitive intelligence $(\mathrm{CI})$ for evaluation of construction contractors. Automation in Construction, 59, 149-157. https://doi.org/10.1016/j. autcon.2015.02.009

Teixeira, R. C., \& Souza, R. R. (2013). O uso das informações contidas em documentos de patentes nas práticas de Inteligência Competitiva: apresentação de um estudo das patentes da UFMG. Perspectivas Em Ciência Da Informação, 18(1), 106-125. https://doi.org/10.1590/ S1413-99362013000100008

Van Eck, N. J., \& Waltman, L. (2010). Software survey: VOSviewer, a computer program for bibliometric mapping. Scientometrics, 84(2), 523-538. https:/doi. org/10.1007/s11192-009-0146-3

Yap, C. S., Rashid, M. Z. A., \& Sapuan, D. A. (2013). Perceived environmental uncertainty and competitive intelligence practices. The Journal of Information and Knowledge Management Systems, 43(4), 462-481. https://doi.org/10.1108/VINE-11-2011-0058

Yin, R. K. (2017). Case study research and applications: Design and methods. Sage publications.

Zupic, I., \& Čater, T. (2015). Bibliometric Methods in Management and Organization. Organizational Research Methods, 18(3), 429-472. https://doi. org/10.1177/1094428114562629

\section{Artigos considerados na Análise Bibliométrica}

1. NG, K.K.H.; TANG, M.H.M.; LEE, C.K.M. Design and development of a performance evaluation system for the aircraft maintenance industry, IEEE INTERNATIONAL CONFERENCE ON INDUSTRIAL ENGINEERING AND ENGINEERING MANAGEMENT, 2016. 
2. SAFA, M.; SHAHI, A.; HAAS, C.T.; FIANDERMCCANN, D.; SAFA, M.; HIPEL, K.; MACGILLIVRAY, S. Competitive intelligence (CI) for evaluation of construction contractors, AUTOMATION IN CONSTRUCTION, 2015.

3. KIM, Y.; KANG, S.; KIM, T.; CHOI, Y.; PARK, M.; CHOI, S. Sentiment analysis of consumer opinion in blogs: A case study in Ramen market, ACM INTERNATIONAL CONFERENCE PROCEEDING SERIES, 2015.

4. LIPIZZI, C.; IANDOLI, L.; RAMIREZ, MARQUEZ, J.E. Extracting and evaluating conversational patterns in social media: A socio-semantic analysis of customers' reactions to the launch of new products using Twitter streams, INTERNATIONAL JOURNAL OF INFORMATION MANAGEMENT, 2015.

5. WANDA, P.; STIAN, SÆ. The Secret of my Success: An exploratory study of Business Intelligence management in the Norwegian Industry, PROCEDIA COMPUTER SCIENCE, 2015.

6. CHUNG, W. BizPro: Extracting and categorizing business intelligence factors from textual news articles, INTERNATIONAL JOURNAL OF INFORMATION MANAGEMENT, 2014.

7. MCBRIDE, N. Business intelligence in magazine distribution, INTERNATIONAL JOURNAL OF INFORMATION MANAGEMENT, 2014.

8. KAY, L.; YOUTIE, J.; SHAPIRA, P. Signs of things to come? What patent submissions by small and medium-sized enterprises say about corporate strategies in emerging technologies, TECHNOLOGICAL FORECASTING AND SOCIAL CHANGE, 2014.

9. HORKOFF, J.; BARONE, D.; JIANG, L.; YU, E.; AMYOT, D.; BORGIDA, A.; MYLOPOULOS, J. Strategic business modeling: Representation and reasoning, SOFTWARE AND SYSTEMS MODELING, 2014.

10. SCHMIDT, R.; MÖHRING, M.; MAIER, S.; PIETSCH, J.; HÄRTING, R.-C. Big data as strategic enabler - Insights from central european enterprises, LECTURE NOTES IN BUSINESS INFORMATION PROCESSING, 2014.

11. PASCHE, E.; GOBEILL, J.; KREIM, O.; OEZDEMIRZAECH, F.; VACHON, T.; LOVIS, C.; RUCH, P. Development and tuning of an original search engine for patent libraries in medicinal chemistry, BMC BIOINFORMATICS, 2014.

12. YAP, C.S.; RASHID, M.Z.A.; SAPUAN, D.A. Perceived environmental uncertainty and competitive intelligence practices, VINE, 2013.

13. MURRAY, M.J.; CHIN, W.W.; ANDERSONFLETCHER, E. Satisfaction with ERP Systems in Supply Chain Operations, SPRINGER PROCEEDINGS IN MATHEMATICS AND STATISTICS, 2013.
14. HOU, C.-K. Investigating factors influencing the adoption of business intelligence systems: An empirical examination of two competing models, INTERNATIONAL JOURNAL OF TECHNOLOGY, POLICY AND MANAGEMENT, 2013.

15. CHUNG, P.-T.; CHUNG, S.H. On data integration and data mining for developing business intelligence, 9TH ANNUAL CONFERENCE ON LONG ISLAND SYSTEMS, APPLICATIONS AND TECHNOLOGY, LISAT 2013, 2013.

16. PICKERING, C.K. Synergizing people, process, and technology to motivate knowledge sharing and collaboration Industry case study, PROCEEDINGS OF THE 2013 INTERNATIONAL CONFERENCE ON COLLABORATION TECHNOLOGIES AND SYSTEMS, CTS 2013, 2013.

17. VUKŠIĆ, V.B.; BACH, M.P.; POPOVIČ, A. Supporting performance management with business process management and business intelligence: A case analysis of integration and orchestration, INTERNATIONAL JOURNAL OF INFORMATION MANAGEMENT, 2013.

18. KEWO, A.; MANEMBU, P.; SENGKEY, C.H. IntelligEnsia for energy sustainability case study: Manado, Indonesia, PROCEEDINGS - UKSIM-AMSS 7TH EUROPEAN MODELLING SYMPOSIUM ON COMPUTER MODELLING AND SIMULATION, EMS 2013, 2013.

19. HOU, C.-K. Measuring the impacts of the integrating information systems on decision-making performance and organisational performance: An empirical study of the Taiwan semiconductor industry, INTERNATIONAL JOURNAL OF TECHNOLOGY, POLICY AND MANAGEMENT, 2013.

20. PHAN, D.D.; SCHMIDT, M.B.; ROTH, L. Natural intelligence applications for business intelligence in online and catalogue retailing firms: A case study, INTERNATIONAL JOURNAL OF INFORMATION SYSTEMS AND CHANGE MANAGEMENT, 2012.

21. WANG, W.; HWANG, D. A case study of a government-sponsored enterprise resource planning project in a Chinese apparel company, INTERNATIONAL JOURNAL OF ENTERPRISE INFORMATION SYSTEMS, 2012.

22. ROHRBECK, R.; GEMÜNDEN, H.G. Corporate foresight: Its three roles in enhancing the innovation capacity of a firm, TECHNOLOGICAL FORECASTING AND SOCIAL CHANGE, 2011.

23. MOLENSKY, L.; KETTER, W.; COLLINS, J.; BLOEMHOF, J.; VAN, DE, KOPPEL, H. Business intelligence gap analysis: A user, supplier and academic perspective, ACM INTERNATIONAL CONFERENCE PROCEEDING SERIES, 2010.

24. REMINGTON, S.; O’DONNELL, P.; ARNOTT, D. Market strategy and innovation in the business intelligence industry: A preliminary study, FRONTIERS IN ARTIFICIAL INTELLIGENCE AND APPLICATIONS, 2010. 
25. DURMU®ÒLU, S.S.; MCNALLY, R.C.; CALANTONE, R.J.; HARMANCIOGLU, N. How elephants learn the new dance when headquarters changes the music: Three case studies on innovation strategy change, JOURNAL OF PRODUCT INNOVATION MANAGEMENT, 2008.

26. ZHU, Y.; LI, Y.; QIAN, Y.; CHEN, J.; CHEN, J. Informization Implementation for Chinese Retailers, TSINGHUA SCIENCE AND TECHNOLOGY, 2008.

27. SADIQ, S.; ZHOU, X.; DENG, K. Research and practice in data quality, LECTURE NOTES IN COMPUTER SCIENCE (INCLUDING SUBSERIES LECTURE NOTES IN ARTIFICIAL INTELLIGENCE AND LECTURE NOTES IN BIOINFORMATICS), 2008.

28. BOSE, R. Competitive intelligence process and tools for intelligence analysis, INDUSTRIAL MANAGEMENT AND DATA SYSTEMS, 2008.

29. CHAN, F.T.S.; CHAN, H.K.; LAU, H.C.W.; IP, R.W.L. Critical success factors in managing global supply chains, INTERNATIONAL JOURNAL OF MANUFACTURING TECHNOLOGY AND MANAGEMENT, 2008.

30. OZORHON, B.; ARDITI, D.; DIKMEN, I.; BIRGONUL, M.T. Implications of culture in the performance of international construction joint ventures, JOURNAL OF CONSTRUCTION ENGINEERING AND MANAGEMENT, 2008.

31. NEIROTTI, P.; CANTAMESSA, M.; PAOLUCCI, E. Do companies with a competitive advantage make better use of IT? Evidence from Italian enterprises, INTERNATIONAL JOURNAL OF TECHNOLOGY MANAGEMENT, 2008.

32. WIXOM, B.H.; WATSON, H.J.; REYNOLDS, A.M.; HOFFER, J.A. Continental airlines continues to soar with business intelligence, INFORMATION SYSTEMS MANAGEMENT, 2008.

33. ARNOTT, D.; PERVAN, G. Eight key issues for the decision support systems discipline, DECISION SUPPORT SYSTEMS, 2008.

34. FENG, M.; TERZIOVSKI, M.; SAMSON, D. Relationship of ISO 9001:2000 quality system certification with operational and business performance: A survey in Australia and New Zealand-based manufacturing and service companies, JOURNAL OF MANUFACTURING TECHNOLOGY MANAGEMENT, 2008.

35. KOIVUNIEMI, J.; EDELMANN, J. Networked innovation management: A framework and case application, PROCEEDINGS OF THE ANNUAL HAWAII INTERNATIONAL CONFERENCE ON SYSTEM SCIENCES, 2007.

36. LI, Z.; YE, J.; ZOU, Y. An empirical study on the effect mechanism of knowledge management on new product development in aviation industry, 2007 INTERNATIONAL CONFERENCE ON WIRELESS COMMUNICATIONS, NETWORKING AND MOBILE COMPUTING, WICOM 2007, 2007.
37. FUCHINO, T.; BATRES, R.; SHIMADA, Y. A knowledge-based approach for the analysis of abnormal situations, LECTURE NOTES IN COMPUTER SCIENCE (INCLUDING SUBSERIES LECTURE NOTES IN ARTIFICIAL INTELLIGENCE AND LECTURE NOTES IN BIOINFORMATICS), 2007.

38. XIA, L.X.X. Supply chain modelling and improvement in telecom industry: A case study, 2006 IEEE INTERNATIONAL CONFERENCE ON INDUSTRIAL INFORMATICS, INDIN'06, 2007.

39. DU, J.; JIAO, Y.-Y.; JIAO, R.J.; KUMAR, A.; MA, M. A case study of obsolete part procurement process reengineering, IEEM 2007: 2007 IEEE INTERNATIONAL CONFERENCE ON INDUSTRIAL ENGINEERING AND ENGINEERING MANAGEMENT, 2007.

40. NI, W.-B. Success criteria of technological incubation in China: Case study in Hong Kong and Hangzhou, PROCEEDINGS OF 2006 INTERNATIONAL CONFERENCE ON MANAGEMENT SCIENCE AND ENGINEERING, ICMSE'06 (13TH), 2007.

41. ICHIJO, K.; KOHLBACHER, F. The Toyota way of global knowledge creation the 'learn local, act global' strategy, INTERNATIONAL JOURNAL OF AUTOMOTIVE TECHNOLOGY AND MANAGEMENT, 2007.

42. MUNDIM, A.P.F. Knowledge management projects valorisation in an automotive company, INTERNATIONAL JOURNAL OF AUTOMOTIVE TECHNOLOGY AND MANAGEMENT, 2007.

43. KLASSEN, R.D.; MENOR, L.J. The process management triangle: An empirical investigation of process trade-offs, JOURNAL OF OPERATIONS MANAGEMENT, 2007.

44. RUSINKO, C.A. Green manufacturing: An evaluation of environmentally sustainable manufacturing practices and their impact on competitive outcomes, IEEE TRANSACTIONS ON ENGINEERING MANAGEMENT, 2007.

45. JOSHI, N.N.; LAMBERT, J.H. Equity metrics with risk, performance, and cost objectives for the prioritization of transportation projects, IEEE TRANSACTIONS ON ENGINEERING MANAGEMENT, 2007.

46. ROBERTSON, P.L.; PATEL, P.R. New wine in old bottles: Technological diffusion in developed economies, RESEARCH POLICY, 2007.

47. DWYER, J. Nissan's supplier struggle with continuous improvement [Automobile industry], MANUFACTURING ENGINEER, 2007.

48. ALBORS, J.; HERVÁS, J.L. CI practice in Spain: Its role as a strategic tool for the firm. Empirical evidence from the CINet survey analysis, INTERNATIONAL JOURNAL OF TECHNOLOGY MANAGEMENT, 2007. 
49. CHOU, Y.-C.; CHENG, C.-T.; YANG, F.-C.; LIANG, Y.-Y. Evaluating alternative capacity strategies in semiconductor manufacturing under uncertain demand and price scenarios, INTERNATIONAL JOURNAL OF PRODUCTION ECONOMICS, 2007.

50. SEO, D.; LEE, J. Gaining competitive advantage through value-shifts: A case of the South Korean wireless communications industry, INTERNATIONAL JOURNAL OF INFORMATION MANAGEMENT, 2007.

51. CHEAH, C.; KANG, J.; CHEW, D. Strategic analysis of large local construction firms in China, CONSTRUCTION MANAGEMENT AND ECONOMICS, 2007.

52. WU, X.; WU, Z. Building competitive advantage with interorganizational information systems in value chain: Evidence from chain retail industry, ICMIT 2006 PROCEEDINGS - 2006 IEEE INTERNATIONAL CONFERENCE ON MANAGEMENT OF INNOVATION AND TECHNOLOGY, 2006.

53. PEÑALOZA, E.; BROOKS, M.; MARCHE, S. Electronic commerce and the strategic management of deep-sea container shipping companies: An exploratory a survey analysis, PROCEEDINGS OF THE ACM CONFERENCE ON ELECTRONIC COMMERCE, 2006.

54. ZHANG, X.-L.; GONG, W.-J.; NARITA, T. Business intelligence in telecommunication enterprises: A case study of log data analysis, PROCEEDINGS OF THE 2006 INTERNATIONAL CONFERENCE ON MACHINE LEARNING AND CYBERNETICS, 2006.

55. MULEBEKE, J.A.W.; ZHENG, L. Analytical network process for software selection in product development: A case study, JOURNAL OF ENGINEERING AND TECHNOLOGY MANAGEMENT - JET-M, 2006.

56. RICHTER, K.; ERNST, R. How OEMs and suppliers can face the network integration challenges, PROCEEDINGS -DESIGN, AUTOMATION AND TEST IN EUROPE, DATE, 2006.

57. JARDIM-GONCALVES, R.; GRILO, A.; STEIGERGARCAO, A. Challenging the interoperability between computers in industry with MDA and SOA, COMPUTERS IN INDUSTRY, 2006.

58. HELAAKOSKI, H.; ISKANIUS, P.; PELTOMAA, I.; KIPINÄ, J. Agile business model in the steel product industry sector, ICMIT 2006 PROCEEDINGS 2006 IEEE INTERNATIONAL CONFERENCE ON MANAGEMENT OF INNOVATION AND TECHNOLOGY, 2006.

59. MOUSAEI, A.; MOGHADDAM, A.A.; GHADIRIAN, A. Developing a model for technology commercialisation of petrochemical products: A case study for knowledge-intensive industries in Research Institute of Petroleum Industry (RIPI), Iran, INTERNATIONAL JOURNAL OF TECHNOLOGY, POLICY AND MANAGEMENT, 2006.
60. LAYMAN, L.; WILLIAMS, L.; CUNNINGHAM, L. Motivations and measurements in an agile case study, JOURNAL OF SYSTEMS ARCHITECTURE, 2006.

61. FU, W.-K.; LO, H.-P.; DREW, D.S. Collective learning, collective knowledge and learning networks in construction, CONSTRUCTION MANAGEMENT AND ECONOMICS, 2006.

62. BULL, L.; FERGUSON, I. Factors influencing the success of wood product innovations in Australia and New Zealand, FOREST POLICY AND ECONOMICS, 2006.

63. GREGOR, S.; MARTIN, M.; FERNANDEZ, W.; STERN, S.; VITALE, M. The transformational dimension in the realization of business value from information technology, JOURNAL OF STRATEGIC INFORMATION SYSTEMS, 2006.

64. MORTON, S.C.; DAINTY, A.R.J.; BURNS, N.D.; BROOKES, N.J.; BACKHOUSE, C.J. Managing relationships to improve performance: A case study in the global aerospace industry, INTERNATIONAL JOURNAL OF PRODUCTION RESEARCH, 2006.

65. HUA, S.Y.; WEMMERLÖV, U. Product change intensity in the personal computer industry: A study of firm-supplier innovation, IEEE TRANSACTIONS ON ENGINEERING MANAGEMENT, 2006.

66. AYLWARD, D. Global pipelines: Profiling successful SME exporters within the Australian wine industry, INTERNATIONAL JOURNAL OF TECHNOLOGY, POLICY AND MANAGEMENT, 2006.

67. MANRING, S.L.; MOORE, S.B. Creating and managing a virtual inter-organizational learning network for greener production: a conceptual model and case study, JOURNAL OF CLEANER PRODUCTION, 2006.

68. MESSNARZ, R.; O'SUILLEABHAIN, G.; COUGHLAN, R. From process improvement to learning organisations, SOFTWARE PROCESS IMPROVEMENT AND PRACTICE, 2006.

69. FJELDSTAD, Ø.D.; KETELS, C.H.M. Competitive Advantage and the Value Network Configuration. Making Decisions at a Swedish Life Insurance Company, LONG RANGE PLANNING, 2006.

70. IBRAHIM, S.; FALLAH, M.H. Where do inventors get their ideas?, PORTLAND INTERNATIONAL CONFERENCE ON MANAGEMENT OF ENGINEERING AND TECHNOLOGY, 2005.

71. ZHENMING, X.; MIA, Z.; XIAODAN, J. Business intelligence - A case study in life insurance industry, PROCEEDINGS - ICEBE 2005: IEEE INTERNATIONAL CONFERENCE ON E-BUSINESS ENGINEERING, 2005.

72. MALHOTRA, A.; MAJCHRZAK, A. Enabling knowledge creation in far-flung teams: Best practices for IT support and knowledge sharing, IEEE ENGINEERING MANAGEMENT REVIEW, 2005. 
73. LICHTENTHALER, E. The choice of technology intelligence methods in multinationals: Towards a contingency approach, INTERNATIONAL JOURNAL OF TECHNOLOGY MANAGEMENT, 2005.

74. SZCZERBICKI, E.; DRINKWATER, M. Concurrent engineering design for environment, CYBERNETICS AND SYSTEMS, 2004.

75. CHOLASUKE, C.; BHARDWA, R.; ANTONY, $\mathrm{J}$. The status of maintenance management in UK manufacturing organisations: Results from a pilot survey, JOURNAL OF QUALITY IN MAINTENANCE ENGINEERING, 2004.

76. $\mathrm{CHO}, \mathrm{Y}$. The organizational boundaries of housebuilding firms in Korea, CONSTRUCTION MANAGEMENT AND ECONOMICS, 2003.

77. JUNG, H.S.; HUR, D.; PARK, J.K. Congestion cost allocation method in a pool model, IEE PROCEEDINGS: GENERATION, TRANSMISSION AND DISTRIBUTION, 2003.

78. VOORDIJK, H.; VAN, LEUVEN, A.; LAAN, A. Enterprise resource planning in a large construction firm: Implementation analysis, CONSTRUCTION MANAGEMENT AND ECONOMICS, 2003.

79. XU, X.M.; KAYE, G.R.; DUAN, Y. UK executives' vision on business environment for information scanning: A cross industry study, INFORMATION AND MANAGEMENT, 2003.

80. NASSIMBENI, G. Local manufacturing systems and global economy: Are they compatible? The case of the Italian eyewear district, JOURNAL OF OPERATIONS MANAGEMENT, 2003.

81. MCKEOWN, I.; PHILIP, G. Business transformation, information technology and competitive strategies: Learning to fly, INTERNATIONAL JOURNAL OF INFORMATION MANAGEMENT, 2003.

82. MOMME, J. Framework for outsourcing manufacturing: Strategic and operational implications, COMPUTERS IN INDUSTRY, 2002.

83. SOHAL, A.S.; POWER, D.J.; TERZIOVSKI, M. Supply chain management in Australian manufacturing - Two case studies, COMPUTERS AND INDUSTRIAL ENGINEERING, 2002.
84. NGOWI, A.B.; IWISI, D.S.; MUSHI, R.J. Competitive strategy in a context of low financial resources, BUILDING RESEARCH AND INFORMATION, 2002.

85. PALMBERG, C. Technological systems and competent procurers - The transformation of Nokia and the Finnish telecom industry revisited?, TELECOMMUNICATIONS POLICY, 2002.

86. HALL, L.A.; BAGCHI-SEN, S. A study of R\&D, innovation, and business performance in the Canadian biotechnology industry, TECHNOVATION, 2002.

87. EFSTATHIADES, A.; TASSOU, S.; ANTONIOU, A. Strategic planning, transfer and implementation of Advanced Manufacturing Technologies (AMT). Development of an integrated process plan, TECHNOVATION, 2002.

88. TANG, V.; LEE, M.-H. International joint venture of two giants in the CRT industry: Strategy analysis using system dynamics, INTERNATIONAL JOURNAL OF TECHNOLOGY MANAGEMENT, 2002.

89. DETIENNE, D.R.; KOBERG, C.S. The impact of environmental and organizational factors on discontinuous innovation within high-technology industries, IEEE TRANSACTIONS ON ENGINEERING MANAGEMENT, 2002.

90. ISKANDAR, B.Y.; KUROKAWA, S.; LEBLANC, L.J. Business-to-business electronic commerce from firstand second-tier automotive suppliers' perspectives: A preliminary analysis for hypotheses generation, TECHNOVATION, 2001.

91. LUREY, J.S.; RAISINGHANI, M.S. An empirical study of best practices in virtual teams, INFORMATION AND MANAGEMENT, 2001.

92. KALAFSKY, R.V.; MACPHERSON, A.D. Recent trends in the export performance of US machine tool companies, TECHNOVATION, 2001.

93. MARTIN, I.; CHEUNG, Y. Change management at mobil oil Australia, PROCEEDINGS OF THE HAWAII INTERNATIONAL CONFERENCE ON SYSTEM SCIENCES, 2001.

Recebido em 4 fev. 2018 / aprovado em 13 mar. 2018

Para referenciar este texto

Azarias, J. G., Coutinho, A. R., \& Campos, F. C. Análise bibliométrica sobre estudo de caso e Survey voltado a inteligência competitiva na indústria. Exacta, São Paulo, v. 16, n. 4, p. 135-148. out./dez. 2018. Disponível em: <https://doi.org/10.5585/ExactaEP. v16n4.8331> 